\title{
The Explosion at the Center of Our Galaxy
}

\section{Kenneth Dalton}

Th. Pongprasart, Bang Saphan, Prachuap Kiri Khan, Thailand

Email:kxdalton@yahoo.com

How to cite this paper: Dalton, K. (2020) The Explosion at the Center of Our Galaxy. Journal of High Energy Physics, Gravitation and Cosmology, 6, 440-442. https://doi.org/10.4236/jhepgc.2020.63033

Received: May 30, 2020

Accepted: July 19, 2020

Published: July 22, 2020

Copyright (c) 2020 by author(s) and Scientific Research Publishing Inc. This work is licensed under the Creative Commons Attribution International License (CC BY 4.0).

http://creativecommons.org/licenses/by/4.0/

\begin{abstract}
At the center of the Milky Way, our black hole may have suddenly changed from supermassive to intermediate-mass status. In doing so, it would have emitted an enormous burst of electromagnetic radiation. Here, the total energy of that burst is calculated and compared with the Fermi bubble data.
\end{abstract}

\section{Keywords}

Black Hole Explosion, Fermi Bubbles

In the electron-positron model [1], black holes naturally separate into two distinct varieties. Those of intermediate mass are supported against gravity by electron degeneracy pressure, while supermassive black holes are supported by ideal gas and radiation pressure. Their equilibrium states are reproduced in Table 1 and Table 2.

A critical mass of $8 \times 10^{6} \mathrm{M}_{\odot}$ defines the transition region.

Over the course of time, these black holes gradually lose energy by heating ionized gas in the accretion disk. They also suffer the loss of electrons, positrons and low-level radiation directly from the black hole itself. This raises the possibility that our black hole $\left(4 \times 10^{6} \mathrm{M}_{\odot}\right.$, Table 1$)$ may have undergone a spontaneous

Table 1. Intermediate-mass.

\begin{tabular}{ccccc}
\hline$M\left(\mathrm{M}_{\odot}\right)$ & $R>R_{s}(\mathrm{~cm})$ & $\rho_{0}\left(\mathrm{~g} \cdot \mathrm{cm}^{-3}\right)$ & $P_{0}(\mathrm{~Pa})$ & $\epsilon_{F 0}(\mathrm{eV})$ \\
\hline $10^{3}$ & $4.8\left(10^{13}\right)$ & $2.6\left(10^{-5}\right)$ & $3.9\left(10^{9}\right)$ & 2.1 \\
$10^{4}$ & $2.25\left(10^{13}\right)$ & $2.6\left(10^{-3}\right)$ & $8.4\left(10^{12}\right)$ & $4.5(10)$ \\
$10^{5}$ & $1.05\left(10^{13}\right)$ & $2.6\left(10^{-1}\right)$ & $1.8\left(10^{16}\right)$ & $9.6\left(10^{2}\right)$ \\
$10^{6}$ & $4.8\left(10^{12}\right)$ & $2.6(10)$ & $3.9\left(10^{19}\right)$ & $2.1\left(10^{4}\right)$ \\
$4\left(10^{6}\right)$ & $3.0\left(10^{12}\right)$ & $4.2\left(10^{2}\right)$ & $4.0\left(10^{20}\right)$ & $1.4\left(10^{5}\right)$ \\
$8\left(10^{6}\right)$ & $2.4\left(10^{12}\right)$ & $1.7\left(10^{3}\right)$ & $3.9\left(10^{22}\right)$ & $3.3\left(10^{5}\right)$ \\
\hline
\end{tabular}


Table 2. Supermassive.

\begin{tabular}{ccccc}
\hline$M\left(\mathrm{M}_{\odot}\right)$ & $R=R_{s}(\mathrm{~cm})$ & $\rho_{0}\left(\mathrm{~g} \cdot \mathrm{cm}^{-3}\right)$ & $P_{0}(\mathrm{~Pa})$ & $k T_{0}(\mathrm{eV})$ \\
\hline $8\left(10^{6}\right)$ & $2.4\left(10^{12}\right)$ & $2\left(10^{3}\right)$ & $3.9\left(10^{22}\right)$ & $1.1\left(10^{5}\right)$ \\
$10^{7}$ & $3\left(10^{12}\right)$ & $1.3\left(10^{3}\right)$ & $2.5\left(10^{22}\right)$ & $1.1\left(10^{5}\right)$ \\
$10^{8}$ & $3\left(10^{13}\right)$ & $1.3(10)$ & $2.5\left(10^{20}\right)$ & $6.2\left(10^{4}\right)$ \\
$10^{9}$ & $3\left(10^{14}\right)$ & $1.3\left(10^{-1}\right)$ & $2.5\left(10^{18}\right)$ & $2.3\left(10^{4}\right)$ \\
$10^{10}$ & $3\left(10^{15}\right)$ & $1.3\left(10^{-3}\right)$ & $2.5\left(10^{16}\right)$ & $8\left(10^{3}\right)$ \\
$10^{11}$ & $3\left(10^{16}\right)$ & $1.3\left(10^{-5}\right)$ & $2.5\left(10^{14}\right)$ & $2\left(10^{3}\right)$ \\
\hline
\end{tabular}

transition from supermassive to intermediate-mass status. The radius of the metastable state would have been $R=R_{s}=1.2 \times 10^{12} \mathrm{~cm}$. At a point in time millions of years ago, the radius suddenly increased to $R=2.5 R_{s}=3 \times 10^{12} \mathrm{~cm}$, releasing the electromagnetic radiation. The remaining leptons settled into the stable quantum state that exists today.

The pressure in a supermassive black hole is given by

$$
P=P_{\mathrm{gas}}+P_{\mathrm{rad}}=\frac{\rho}{m} k T+\frac{a}{3} T^{4}
$$

where $a=\pi^{2} k^{4} / 15(\hbar c)^{3}$. In states near the transition region, the gas pressure is far greater than the radiation pressure. This is due to the high number density of leptons. For purposes of calculation, it is simpler to adopt the uniform density model [2], in which case the pressure satisfies

$$
P=P_{0}\left(1-\frac{r^{2}}{R^{2}}\right)
$$

Ignoring the radiation pressure leaves the linear ideal gas relation between pressure and temperature, so that

$$
T=T_{0}\left(1-\frac{r^{2}}{R^{2}}\right)
$$

The energy density of radiation is

$$
u_{\mathrm{rad}}=a T^{4}=a T_{0}^{4}\left(1-\frac{r^{2}}{R^{2}}\right)^{4}
$$

which yields the total radiant energy

$$
U_{\mathrm{rad}}=\int_{0}^{R} u_{\mathrm{rad}} 4 \pi r^{2} \mathrm{~d} r=1.6 \times 10^{58} \mathrm{erg}
$$

where $M=4 \times 10^{6} \mathrm{M}_{\odot}, R=1.2 \times 10^{12} \mathrm{~cm}$ and $T_{0}=1.3 \times 10^{9} \mathrm{~K}$.

The energy calculated here is an order of magnitude greater than the current upper estimates for the Fermi bubbles. Nevertheless, given the experimental uncertainties and given the limitations of the model, it may be said that the work is in substantial agreement with observation. 


\section{Conflicts of Interest}

The author declares no conflicts of interest regarding the publication of this paper.

\section{References}

[1] Dalton, K. (2019) Supermassive Black Holes. JHEPGC, 5, 984-988. https://doi.org/10.4236/jhepgc.2019.53052

[2] Dalton, K. (2014) The Galactic Black Hole. Hadronic J., 37, 241. https://vixra.org/abs/1404.0067 